\title{
MIXED OF ELZAKI TRANSFORM AND PROJECTED DIFFERENTIAL TRANSFORM METHOD FOR A NONLINEAR WAVE-LIKE EQUATIONS WITH VARIABLE COEFFICIENTS
}

\author{
A. KHALOUTA ${ }^{1}$ AND A. KADEM ${ }^{2}$
}

\begin{abstract}
In this work, a mixture of Elzaki transform and projected differential transform method is applied to solve a nonlinear wave-like equations with variable coefficients. Nonlinear terms can be easily manipulated by using the projected differential transformation method. The method gives the results show that the proposed method is very efficient, simple and can be applied to other applications.
\end{abstract}

\section{Introduction}

The integral transforms play a significant role in many fields of science and in the literature, it's are largely used in mathematical physics, optics, mathematical engineering and in some others in order to solve the differential equations such as Laplace, Fourier, Mellin, Hankel and Sumudu.

Recently, Tarig Elzaki [5] introduced a new integral transform, called Elzaki transform, which is applied to solve an ordinary and partial differential equations.

By applying the Adomian decomposition method (ADM), M. Ghoreishi solved some types of nonlinear wave-like equation [6], V.G. Gupta and S. Gupta worked out by using homotopy perturbation transform method ( HPTM) these types of equation tool [7, furthermore, A. Aslanov [3], F. Yin and et al [12] and A. Atangana and et al [2] researched for solving nonlinear heat and wave-like equation by using homotopy perturbation, variational iteration and homotopy decomposition methods respectively. Moreover, various techniques, such as homotopy analysis, perturbations, decompositions, iterations, differential and Laplace transformation techniques have been used to handle similar types of these wave-like and also heat-like problems numerically and analytically as in references [1, [7],[10,,[11].

In this work, we will present the mixed of Elzaki transform and projected differential transform method, in order to solve a nonlinear wave-like equations. This method called Elzaki projected differential transform method (EPDTM).

2010 Mathematics Subject Classification. Primary 35L05, 35L10; Secondary 35A22, $35 \mathrm{~A} 35$.

Key words and phrases. Nonlinear wave-like equations, Elzaki transform, Projected differential transform method. 
Consider the following nonlinear wave-like equations

$$
\begin{aligned}
\frac{\partial^{2} u}{\partial t^{2}}= & \sum_{i, j=1}^{n} F_{1 i j}(X, t, u) \frac{\partial^{k+m}}{\partial x_{i}^{k} \partial x_{j}^{m}} F_{2 i j}\left(u_{x_{i}}, u_{x_{j}}\right) \\
& +\sum_{i=1}^{n} G_{1 i}(X, t, u) \frac{\partial^{p}}{\partial x_{i}^{p}} G_{2 i}\left(u_{x_{i}}\right)+H(X, t, u)+S(X, t),
\end{aligned}
$$

with the initial conditions

$$
u(X, 0)=a_{0}(X), u_{t}(X, 0)=a_{1}(X) .
$$

Here $X=\left(x_{1}, x_{2}, \ldots, x_{n}\right), F_{1 i j}, G_{1 i} i, j \in\{1,2, \ldots, n\}$ are nonlinear functions of $X, t$ and $u, F_{2 i j}, G_{2 i} i, j \in\{1,2, \ldots, n\}$, are nonlinear functions of derivatives of $u$ with respect to $x_{i}$ and $x_{j} i, j \in\{1,2, \ldots, n\}$, respectively. Also $H, S$ are nonlinear functions and $k, m, p$ are integers.

These types of equations are of considerable significance in various fields of applied sciences, mathematical physics, nonlinear hydrodynamics, engineering physics, biophysics, human movement sciences, astrophysics and plasma physics. These equations describe the evolution of erratic motions of small particles that are immersed in fluids, fluctuations of the intensity of laser light, velocity distributions of fluid particles in turbulent flows.

\section{Elzaki transform}

Definition 2.1. A new integral transform called Elzaki transform [4, [5] defined for functions of exponential order, is proclaimed. We consider functions in the set $A$ defined by,

$$
A=\left\{f(t) / \exists M, k_{1}, k_{2}>0,|f(t)|<M e^{\frac{|t|}{k_{j}}}, \text { if } t \in(-1)^{j} \times[0, \infty)\right\} .
$$

Elzaki transform of the function $f(t)$ is

$$
E[f(t)]=T(v)=v \int_{0}^{\infty} f(t) e^{-\frac{t}{v}} d t, t>0,
$$

where $v$ is the factor of variable $t$.

T. M. Elzaki and S. M. Elzaki in [4], showed the modified of Sumudu transform [9] or Elzaki transform was applied to partial differential equations, ordinary differential equations, system of ordinary and partial differential equations and integral equations.

Proposition 2.2. To obtain Elzaki transform of partial derivative we use integration by parts and then we have

$$
\begin{aligned}
E\left[\frac{\partial f(x, t)}{\partial t}\right] & =\frac{1}{v} T(x, v)-v f(x, 0) \\
E\left[\frac{\partial f(x, t)}{\partial x}\right] & =\frac{d}{d x}[T(x, v)] \\
E\left[\frac{\partial^{2} f(x, t)}{\partial t^{2}}\right] & =\frac{1}{v^{2}} T(x, v)-f(x, 0)-v \frac{\partial f(x, 0)}{\partial t}, \\
E\left[\frac{\partial^{2} f(x, t)}{\partial x^{2}}\right] & =\frac{d^{2}}{d x^{2}}[T(x, v)] .
\end{aligned}
$$


Proof.

$$
\begin{aligned}
E\left[\frac{\partial f(x, t)}{\partial t}\right] & =\int_{0}^{\infty} v \frac{\partial f}{\partial t} e^{-\frac{t}{v}} d t=\lim _{p \rightarrow \infty} \int_{0}^{p} v e^{-\frac{t}{v}} \frac{\partial f}{\partial t} d t \\
& =\lim _{p \rightarrow \infty}\left\{\left[v e^{-\frac{t}{v}} f(x, t)\right]_{0}^{p}-\int_{0}^{p} e^{-\frac{t}{v}} f(x, t) d t\right\} \\
& =\frac{1}{v} T(x, v)-v f(x, 0) .
\end{aligned}
$$

We assume that $f$ is piecewise continuous and it is of exponential order.

Now

$$
E\left[\frac{\partial f(x, t)}{\partial x}\right]=\int_{0}^{\infty} v e^{-\frac{t}{v}} \frac{\partial f(x, t)}{\partial x} d t=\frac{\partial}{\partial x} \int_{0}^{\infty} v e^{-\frac{t}{v}} f(x, t) d t
$$

using the Leibniz rule to find

$$
E\left[\frac{\partial f(x, t)}{\partial x}\right]=\frac{d}{d x}[T(x, v)] .
$$

By the same method we find

$$
E\left[\frac{\partial^{2} f(x, t)}{\partial x^{2}}\right]=\frac{d^{2}}{d x^{2}}[T(x, v)]
$$

and

$$
E\left[\frac{\partial^{2} f(x, t)}{\partial t^{2}}\right]
$$

Let

$$
\frac{\partial f}{\partial t}=g
$$

then we have

$$
\begin{aligned}
E\left[\frac{\partial^{2} f(x, t)}{\partial t^{2}}\right] & =E\left[\frac{\partial g(x, t)}{\partial t}\right]=\frac{1}{v} E[g(x, t)]-v g(x, 0) \\
& =\frac{1}{v^{2}} T(x, v)-f(x, 0)-v \frac{\partial f(x, 0)}{\partial t} .
\end{aligned}
$$

We can easily extend this result to the $n$th partial derivative by using mathematical induction, we have

$$
E\left[\frac{\partial^{n} f(x, t)}{\partial t^{n}}\right]=\frac{1}{v^{n}} T(x, v)-\sum_{k=0}^{n-1} v^{2-n+k} \frac{\partial^{k} f(x, 0)}{\partial t^{k}} .
$$

The proof is complete.

Properties of Elzaki transform can be found in [4, [5] we mention only the following

$$
\begin{aligned}
E(1) & =v^{2} \\
E(t) & =v^{3} \\
E\left(t^{n}\right) & =n ! v^{n+2} .
\end{aligned}
$$




\section{Projected differential transform method}

In this section, we introduce the basic idea of modified version of the differential transform method (DTM), the projected differential transform method (PDTM) [8. The DTM is based on the Taylor series for all variables. Here, we consider the Talyor series of the function $u$ with respect to the specific variable. Assume that the specific variable is the variable $t$.

Definition 3.1. The projected differential transform $U(X, k)$ of $u(X, t)$ with respect to the variable $t$ at $t_{0}$ is defined by

$$
U(X, k)=\frac{1}{k !}\left[\frac{\partial^{k}}{\partial t^{k}} u(X, t)\right]_{t=t_{0}}
$$

where $X=\left(x_{1}, x_{2}, \ldots, x_{n}\right), u(X, t)$ is the original function and $U(X, k)$ is the transformed function of $u(X, t)$.

Definition 3.2. The projected differential inverse transform of $U(X, k)$ with respect to the variable $t$ at $t_{0}$ is defined by

$$
u(X, t)=\sum_{k=0}^{\infty} U(X, k)\left(t-t_{0}\right)^{k}
$$

Combining Eqs. (3.1) and (3.2), we have the Taylor series expansion of the function $u$ at $t=t_{0}$ as follows

$$
u(X, t)=\sum_{k=0}^{\infty} \frac{1}{k !}\left[\frac{\partial^{k}}{\partial t^{k}} u(X, t)\right]_{t=t_{0}}\left(t-t_{0}\right)^{k} .
$$

From the above definitions, the fundamental operations of the PDTM are given by the following theorems

Theorem 3.3. Let $U(X, k), W(X, k)$ and $Z(X, k)$ be the projected differential transform of the functions $u(X, t), w(X, t)$ and $z(X, t)$ respectively, where $X=\left(x_{1}, x_{2}, \ldots, x_{n}\right)$, then

(1) if

$$
z(X, t)=\lambda u(X, t)+\mu w(X, t),
$$

then

$$
Z(X, k)=\lambda U(X, k)+\mu W(X, k),
$$

where $\lambda$ and $\mu$ are constants.

(2) if

$$
z(X, t)=u(X, t) w(X, t),
$$

then

$$
Z(X, k)=\sum_{r=0}^{k} U(X, r) W(X, k-r)=\sum_{r=0}^{k} W(X, r) U(X, k-r) .
$$

(3) if

$$
z(X, t)=u_{1}(X, t) u_{2}(X, t) \ldots u_{n}(X, t),
$$


then

$$
\begin{aligned}
Z(X, k)= & \sum_{k_{n-1}=0}^{k} \sum_{k_{n-2}=0}^{k_{n-1}} \ldots \sum_{k_{2}=0}^{k_{3}} \sum_{k_{1}=0}^{k_{2}} U_{1}\left(X, k_{1}\right) U_{2}\left(X, k_{2}-k_{1}\right) \\
& \times \ldots \times U_{n-1}\left(X, k_{n-1}-k_{n-2}\right) U_{n}\left(X, k-k_{n-1}\right) .
\end{aligned}
$$

(4) if

$z(X, t)=\frac{\partial^{n}}{\partial t^{n}} u(X, t)$,

then

$$
\begin{aligned}
Z(X, k) & =(k+1)(k+2) \ldots(k+n) U(X, k+n) \\
& =\frac{(k+n) !}{k !} U(X, k+n), n \in\{1,2, \ldots\} .
\end{aligned}
$$

(5) if

$z(X, t)=\frac{\partial^{n}}{\partial x_{i}^{n}} u\left(x_{1}, x_{1}, \ldots, x_{n}, t\right)$,

then

$$
\begin{aligned}
Z(X, k) & =\frac{\partial^{n}}{\partial x_{i}^{n}} U\left(x_{1}, x_{1}, \ldots, x_{n}, k\right) \\
i & \in\{1,2, \ldots, n\}, n \in\{1,2, \ldots\}
\end{aligned}
$$

(6) if

$$
z(X, t)=x_{1}^{a_{1}} x_{2}^{a_{2}} \ldots x_{n}^{a_{n}} t^{a_{m}},
$$

then

$$
Z(X, k)=x_{1}^{a_{1}} x_{2}^{a_{2}} \ldots x_{n}^{a_{n}} \delta\left(k_{m}-a_{m}\right)=\left\{\begin{array}{c}
x_{1}^{a_{1}} x_{2}^{a_{2}} \ldots x_{n}^{a_{n}}, k_{m}=a_{m} \\
0, \text { otherwise }
\end{array} .\right.
$$

(7) if

$z(X, t)=x_{1}^{a_{1}} x_{2}^{a_{2}} \ldots x_{n}^{a_{n}} t^{a_{m}} u(X, t)$,

then

$Z(X, k)=x_{1}^{a_{1}} x_{2}^{a_{2}} \ldots x_{n}^{a_{n}} U(X, k-n)$.

4. EPDTM FOR NONLINEAR WAVE-LIKE EQUATIONS

In this section we describe the application of the Elzaki projected differential transform method (EPDTM) for nonlinear wave-like equations with initial conditions.

Theorem 4.1. Consider the nonlinear wave-like equations

$$
\begin{aligned}
\frac{\partial^{2} u}{\partial t^{2}}= & \sum_{i, j=1}^{n} F_{1 i j}(X, t, u) \frac{\partial^{k+m}}{\partial x_{i}^{k} \partial x_{j}^{m}} F_{2 i j}\left(u_{x_{i}}, u_{x_{j}}\right) \\
& +\sum_{i=1}^{n} G_{1 i}(X, t, u) \frac{\partial^{p}}{\partial x_{i}^{p}} G_{2 i}\left(u_{x_{i}}\right)+H(X, t, u)+S(X, t),
\end{aligned}
$$

with the initial conditions

(4.2) $\quad u(X, 0)=a_{0}(X), u_{t}(X, 0)=a_{1}(X)$. 
Here $X=\left(x_{1}, x_{2}, \ldots, x_{n}\right), F_{1 i j}, G_{1 i} i, j \in\{1,2, \ldots, n\}$ are nonlinear functions of $X, t$ and $u, F_{2 i j}, G_{2 i} i, j \in\{1,2, \ldots, n\}$, are nonlinear functions of derivatives of $u$ with respect to $x_{i}$ and $x_{j} i, j \in\{1,2, \ldots, n\}$, respectively. Also $H, S$ are nonlinear functions and $k, m, p$ are integers.

Then by EPDTM we have the solution of Eqs. (4.1) with initial condition (4.2) in the form of infinite series which converge rapidly to the exact solution as follows

$$
u(X, t)=\sum_{k=0}^{\infty} U(X, k),
$$

where $U(X, k)$ is projected transform function of $u(X, t)$.

Proof. In order to to achieve our goal, we consider the following nonlinear wave-like Eqs. (4.1) with the initial conditions 4.2 .

First, we take the Elzaki transform on both sides of (4.1) subject to initial conditions 4.2), we get

$$
\begin{aligned}
E\left[\frac{\partial^{2} u}{\partial t^{2}}\right]= & E\left[\sum_{i, j=1}^{n} F_{1 i j}(X, t, u) \frac{\partial^{k+m}}{\partial x_{i}^{k} \partial x_{j}^{m}} F_{2 i j}\left(u_{x_{i}}, u_{x_{j}}\right)\right. \\
& \left.+\sum_{i=1}^{n} G_{1 i}(X, t, u) \frac{\partial^{p}}{\partial x_{i}^{p}} G_{2 i}\left(u_{x_{i}}\right)+H(X, t, u)\right]+E[S(X, t)] .
\end{aligned}
$$

Using the differentiation property of Elzaki transforms 2.2 and above initial conditions, we have

$$
\begin{aligned}
E[u(X, t)]= & v^{2} u(X, 0)+v^{3} u_{t}(X, 0)+v^{2} E[S(X, t)] \\
& +v^{2} E\left[\sum_{i, j=1}^{n} F_{1 i j}(X, t, u) \frac{\partial^{k+m}}{\partial x_{i}^{k} \partial x_{j}^{m}} F_{2 i j}\left(u_{x_{i}}, u_{x_{j}}\right)\right. \\
& \left.+\sum_{i=1}^{n} G_{1 i}(X, t, u) \frac{\partial^{p}}{\partial x_{i}^{p}} G_{2 i}\left(u_{x_{i}}\right)+H(X, t, u)\right] .
\end{aligned}
$$

Applying the inverse Elzaki transform on both sides of Eq. (4.4), to find

$$
\begin{aligned}
u(X, t)= & L(X, t)+E^{-1}\left(v ^ { 2 } E \left[\sum_{i, j=1}^{n} F_{1 i j}(X, t, u) \frac{\partial^{k+m}}{\partial x_{i}^{k} \partial x_{j}^{m}} F_{2 i j}\left(u_{x_{i}}, u_{x_{j}}\right)\right.\right. \\
(4.5) \quad & \left.\left.+\sum_{i=1}^{n} G_{1 i}(X, t, u) \frac{\partial^{p}}{\partial x_{i}^{p}} G_{2 i}\left(u_{x_{i}}\right)+H(X, t, u)\right]\right),
\end{aligned}
$$

where

$$
\begin{aligned}
L(X, t) & =E^{-1}\left(v^{2} u(X, 0)+v^{3} u_{t}(X, 0)+v^{2} E[S(X, t)]\right) \\
& =a_{0}(X)+t a_{1}(X)+E^{-1}\left(v^{2} E[S(X, t)]\right)
\end{aligned}
$$

represents the term arising from the source term and the prescribed initial conditions.

Now, we apply the projected differential transform method.

$$
U(X, 0)=L(X, t),
$$




$$
U(X, k+1)=E^{-1}\left(v^{2} E[A(X, k)+B(X, k)+C(X, k)]\right),
$$

where $A(X, k), B(X, k)$ and $C(X, k)$ are the projected differential transformed form of the nonlinear terms of $\sum_{i, j=1}^{n} F_{1 i j}(X, t, u) \frac{\partial^{k+m}}{\partial x_{i}^{k} \partial x_{j}^{m}} F_{2 i j}\left(u_{x_{i}}, u_{x_{j}}\right)$, $\sum_{i=1}^{n} G_{1 i}(X, t, u) \frac{\partial^{p}}{\partial x_{i}^{p}} G_{2 i}\left(u_{x_{i}}\right)$, and $H(X, t, u)$.

From Eq. (4.6), we have

$$
\begin{aligned}
& U(X, 0)=L(X, t), \\
& U(X, 1)=E^{-1}\left(v^{2} E[A(X, 0)+B(X, 0)+C(X, 0)]\right), \\
& U(X, 2)=E^{-1}\left(v^{2} E[A(X, 1)+B(X, 1)+C(X, 1)]\right), \\
& U(X, 3)=E^{-1}\left(v^{2} E[A(X, 2)+B(X, 2)+C(X, 2)]\right),
\end{aligned}
$$

and so on.

Then, the solution of Eqs. 4.1) and (4.2) is given as follows

$$
u(X, t)=\sum_{k=0}^{\infty} U(X, k) .
$$

The proof is complete.

To illustrate the capability and simplicity of the method, some examples for nonlinear partial differential equations will be discussed in particular nonlinear wave-like equations.

\section{Numerical Application}

In this section, we apply mixture of Elzaki transform and the projected differential transform method for solving various types of nonlinear wavelike equations with variable coefficients and we compare the approximate analytical solution obtained for our nonlinear wave-like problems with known exact solutions .

Example 1 Consider the 2-dimensional nonlinear wave-like equation with variable coefficients

$$
\frac{\partial^{2} u}{\partial t^{2}}=\frac{\partial^{2}}{\partial x \partial y}\left(u_{x x} u_{y y}\right)-\frac{\partial^{2}}{\partial x \partial y}\left(x y u_{x} u_{y}\right)-u
$$

with initial conditions

$$
u(x, y, 0)=e^{x y}, u_{t}(x, y, 0)=e^{x y},
$$

where $u=u(x, y, t)$ is a field function, $(x, y, t) \in \mathbb{R} \times \mathbb{R} \times \mathbb{R}^{+}$.

The exact solution to (5.1) with initial conditions (5.2) is given by

$$
u(x, y, t)=e^{x y}(\sin t+\cos t) .
$$

By taking Elzaki transformon on both sides of (5.1) subject to initial conditions (5.2), and using the proposition 2.2. we obtain

$$
E[u(x, y, t)]=v^{2} e^{x y}+v^{3} e^{x y}
$$




$$
+v^{2} E\left[\frac{\partial^{2}}{\partial x \partial y}\left(u_{x x} u_{y y}\right)-\frac{\partial^{2}}{\partial x \partial y}\left(x y u_{x} u_{y}\right)-u\right] \text {. }
$$

By applying the inverse Elzaki transform for (5.3), we get

$$
\begin{aligned}
u(x, y, t)= & e^{x y}+t e^{x y} \\
& +E^{-1}\left(v^{2} E\left[\frac{\partial^{2}}{\partial x \partial y}\left(u_{x x} u_{y y}\right)-\frac{\partial^{2}}{\partial x \partial y}\left(x y u_{x} u_{y}\right)-u\right]\right) .
\end{aligned}
$$

Applying projected differential transform method to obtain

$$
U(x, y, k+1)=E^{-1}\left(v ^ { 2 } E \left[\frac{\partial^{2}}{\partial x \partial y} A(x, y, k)-\frac{\partial^{2}}{\partial x \partial y} x y B(x, y, k)\right.\right.
$$

with

$$
U(x, y, 0)=e^{x y}+t e^{x y},
$$

where $A(x, y, k)$ and $B(x, y, k)$ are the projected differential transformed of the nonlinear terms, $u_{x x} u_{y y}$ and $u_{x} u_{y}$ having the value

$$
\begin{aligned}
& A(x, y, k)=\sum_{r=0}^{k} \frac{\partial^{2} U(x, y, r)}{\partial x^{2}} \frac{\partial^{2} U(x, y, k-r)}{\partial y^{2}} \\
& B(x, y, k)=\sum_{r=0}^{k} \frac{\partial U(x, y, r)}{\partial x} \frac{\partial U(x, y, k-r)}{\partial y} .
\end{aligned}
$$

The few nonlinear terms are as follows

$$
\begin{aligned}
& A(0)=U_{x x}(0) U_{y y}(0) \\
& A(1)=U_{x x}(0) u_{y y}(1)+U_{x x}(1) U_{y y}(0) \\
& A(2)=u_{x x}(0) u_{y y}(2)+u_{x x}(1) u_{y y}(1)+u_{x x}(2) u_{y y}(0)
\end{aligned}
$$

and so on.

$$
\begin{aligned}
& B(0)=U_{x}(0) U_{y}(0) \\
& B(1)=U_{x}(0) U_{y}(1)+U_{x}(1) U_{y}(0) \\
& B(2)=U_{x}(0) u_{y}(2)+U_{x}(1) u_{y}(1)+U_{x}(2) U_{y}(0)
\end{aligned}
$$

and so on.

From the relationship in (5.4), (5.5), we obtain

$$
\begin{aligned}
U(x, y, 0) & =e^{x y}+t e^{x y}=(1+t) e^{x y} \\
U(x, y, 1) & =E^{-1}\left(v^{2} E\left[\frac{\partial^{2}}{\partial x \partial y} A(x, y, 0)-\frac{\partial^{2}}{\partial x \partial y} x y B(x, y, 0)-u(x, y, 0)\right]\right) \\
& =\left(-\frac{t^{2}}{2 !}-\frac{t^{3}}{3 !}\right) e^{x y} \\
U(x, y, 2) & =E^{-1}\left(v^{2} E\left[\frac{\partial^{2}}{\partial x \partial y} A(x, y, 1)-\frac{\partial^{2}}{\partial x \partial y} x y B(x, y, 1)-u(x, y, 1)\right]\right)
\end{aligned}
$$




$$
\begin{aligned}
= & \left(\frac{t^{4}}{4 !}+\frac{t^{5}}{5 !}\right) e^{x y} \\
U(x, y, 3)= & E^{-1}\left(v^{2} E\left[\frac{\partial^{2}}{\partial x \partial y} A(x, y, 2)-\frac{\partial^{2}}{\partial x \partial y} x y B(x, y, 2)-u(x, y, 2)\right]\right) \\
= & \left(-\frac{t^{6}}{6 !}-\frac{t^{7}}{7 !}\right) e^{x y}, \\
& \ldots,
\end{aligned}
$$

and so on.

Which in closed form gives exact solution

$$
\begin{aligned}
u(x, y, t) & =\sum_{k=0}^{\infty} U(x, y, k)=\left(1+t-\frac{t^{2}}{2 !}-\frac{t^{3}}{3 !}+\frac{t^{4}}{4 !}+\frac{t^{5}}{5 !}-\frac{t^{6}}{6 !}-\frac{t^{7}}{7 !}+\ldots\right) e^{x y} \\
& =(\sin t+\cos t) e^{x y} .
\end{aligned}
$$

which is an exactly the same solution obtained by Adomian decomposition method [6] and Homotopy perturbation transform method [7] for the same test problem.

Example 2 Consider the following nonlinear wave-like equation with variable coefficients

$$
\frac{\partial^{2} u}{\partial t^{2}}=u^{2} \frac{\partial^{2}}{\partial x^{2}}\left(u_{x} u_{x x} u_{x x x}\right)+u_{x}^{2} \frac{\partial^{2}}{\partial x^{2}}\left(u_{x x}^{3}\right)-18 u^{5}+u
$$

with initial conditions

$$
u(x, 0)=e^{x}, u_{t}(x, 0)=e^{x},
$$

where $u=u(x, t)$ is a field function, $(x, t) \in] 0,1\left[\times \mathbb{R}^{+}\right.$.

The exact solution to (5.6) with initial conditions (5.7) is given by

$$
u(x, t)=e^{x+t} .
$$

By taking Elzaki transform on both sides of (5.6) subject to initial conditions (5.7), and using the proposition 2.2, we obtain

$$
\begin{aligned}
E[u(x, t)]= & v^{2} e^{x}+v^{3} e^{x} \\
& +v^{2} E\left[u^{2} \frac{\partial^{2}}{\partial x^{2}}\left(u_{x} u_{x x} u_{x x x}\right)+u_{x}^{2} \frac{\partial^{2}}{\partial x^{2}}\left(u_{x x}^{3}\right)-18 u^{5}+u\right]
\end{aligned}
$$

By applying the inverse Elzaki transform for (5.6), we get

$$
\begin{aligned}
u(x, t)= & e^{x}+t e^{x} \\
& +E^{-1}\left[v^{2} E\left[u^{2} \frac{\partial^{2}}{\partial x^{2}}\left(u_{x} u_{x x} u_{x x x}\right)+u_{x}^{2} \frac{\partial^{2}}{\partial x^{2}}\left(u_{x x}^{3}\right)-18 u^{5}+u\right]\right] .
\end{aligned}
$$

Applying projected differential transform method to obtain

$$
U(x, k+1)=E^{-1}\left[v^{2} E[A(x, k)+B(x, k)-18 C(x, k)+u(x, k)]\right],
$$

with

$$
U(x, 0)=e^{x}+t e^{x}
$$

where $A(x, k), B(x, k)$ and $C(x, k)$ are the projected differential transformed of the nonlinear terms $u^{2} \frac{\partial^{2}}{\partial x^{2}}\left(u_{x} u_{x x} u_{x x x}\right), u_{x}^{2} \frac{\partial^{2}}{\partial x^{2}}\left(u_{x x}^{3}\right)$, and $u^{5}$ having the value 


$$
\begin{aligned}
A(x, k)= & \sum_{r=0}^{k} \sum_{s=0}^{r} \sum_{m=0}^{s} \sum_{n=0}^{m} U(x, n) U(x, m-n) \\
& \times \frac{\partial^{2}}{\partial x^{2}}\left[\frac{\partial U(x, s-m)}{\partial x} \frac{\partial^{2} U(x, r-s)}{\partial x^{2}} \frac{\partial^{3} U(x, k-r)}{\partial x^{3}}\right], \\
B(x, k)= & \sum_{r=0}^{k} \sum_{s=0}^{r} \sum_{m=0}^{s} \sum_{n=0}^{m} \frac{\partial U(x, n)}{\partial x} \frac{\partial U(x, m-n)}{\partial x} \\
& \times \frac{\partial^{2}}{\partial x^{2}}\left[\frac{\partial^{2} U(x, s-m)}{\partial x^{2}} \frac{\partial^{2} U(x, r-s)}{\partial x^{2}} \frac{\partial^{2} U(x, k-r)}{\partial x^{2}}\right], \\
C(x, k)= & \sum_{r=0}^{k} \sum_{s=0}^{r} \sum_{m=0}^{s} \sum_{n=0}^{m} U(x, n) U(x, m-n) U(x, s-m) \\
& \times U(x, r-s) U(x, k-r) .
\end{aligned}
$$

The few nonlinear terms are as follows

$$
\begin{aligned}
A(0)= & U^{2}(0) \frac{\partial^{2}}{\partial x^{2}}\left[U_{x}(0) U_{x x}(0) U_{x x x}(0)\right] \\
A(1)= & 2 U(0) U(1) \frac{\partial^{2}}{\partial x^{2}}\left[U_{x}(0) U_{x x}(0) U_{x x x}(0)\right]+U^{2}(0) \frac{\partial^{2}}{\partial x^{2}}\left[U_{x}(1) U_{x x}(0) U_{x x x}(0)\right. \\
& \left.+U_{x}(0) U_{x x}(1) U_{x x x}(0)+U_{x}(0) U_{x x}(0) U_{x x x}(1)\right]
\end{aligned}
$$

and so on.

$$
\begin{aligned}
& B(0)=U_{x}^{2}(0) \frac{\partial^{2}}{\partial x^{2}} U_{x x}^{3}(0) \\
& B(1)=2 U_{x}(0) U_{x}(1) \frac{\partial^{2}}{\partial x^{2}} U_{x x}^{3}(0)+3 U_{x}^{2}(0) \frac{\partial^{2}}{\partial x^{2}}\left[U_{x x}^{2}(0) U_{x x}(1)\right]
\end{aligned}
$$

and so on.

$$
C(0)=U^{5}(0), C(1)=5 U^{4}(0) U(1), \ldots .
$$

and so on.

From the relationship in (5.8), (5.9), we obtain

$$
\begin{aligned}
U(x, 0) & =e^{x}+t e^{x}=(1+t) e^{x} \\
U(x, 1) & =E^{-1}\left[v^{2} E[A(x, 0)+B(x, 0)-18 C(x, 0)+u(x, 0)]\right] \\
= & \left(\frac{t^{2}}{2 !}+\frac{t^{3}}{3 !}\right) e^{x}, \\
U(x, 2)= & E^{-1}\left[v^{2} E[A(x, 1)+B(x, 1)-18 C(x, 1)+u(x, 1)]\right] \\
= & \left(\frac{t^{4}}{4 !}+\frac{t^{5}}{5 !}\right) e^{x},
\end{aligned}
$$

and so on. 
Which in closed form gives exact solution

$$
\begin{aligned}
u(x, t) & =\sum_{k=0}^{\infty} U(x, k)=\left(1+t+\frac{t^{2}}{2 !}+\frac{t^{3}}{3 !}+\frac{t^{4}}{4 !}+\frac{t^{5}}{5 !}+\ldots\right) e^{x} \\
& =e^{x+t}
\end{aligned}
$$

which is an exactly the same solution obtained by Adomian decomposition method [6] and Homotopy perturbation transform method [7] for the same test problem.

Example 3 Consider the following one dimensional nonlinear wave-like equation with variable coefficients

$$
\frac{\partial^{2} u}{\partial t^{2}}=x^{2} \frac{\partial}{\partial x}\left(u_{x} u_{x x}\right)-x^{2}\left(u_{x x}^{2}\right)-u
$$

with initial conditions

$$
u(x, 0)=0, u_{t}(x, 0)=x^{2}
$$

where $u=u(x, t)$ is a field function, $(x, t) \in] 0,1\left[\times \mathbb{R}^{+}\right.$.

The exact solution to (5.10) with initial conditions (5.11) is given by

$$
u(x, t)=x^{2} \sin t .
$$

By taking Elzaki transform on both sides of (5.10) subject to initial conditions (5.11), and using the proposition 2.2, we obtain

$$
E[u(x, t)]=v^{3} x^{2}+v^{2} E\left[x^{2} \frac{\partial}{\partial x}\left(u_{x} u_{x x}\right)-x^{2}\left(u_{x x}^{2}\right)-u\right] .
$$

By applying the inverse Elzaki transform for (5.12), we get

$$
u(x, t)=t x^{2}+E^{-1}\left[v^{2} E\left[x^{2} \frac{\partial}{\partial x}\left(u_{x} u_{x x}\right)-x^{2}\left(u_{x x}^{2}\right)-u\right]\right] .
$$

Applying projected differential transform method to obtain

$$
u(x, k+1)=E^{-1}\left[v^{2} E\left[x^{2} \frac{\partial}{\partial x} A(x, k)-x^{2} B(x, k)-u(x, m)\right]\right],
$$

with

(5.14) $U(x, 0)=t x^{2}$

where $A(k)$ and $B(k)$ are the projected differential transformed of the nonlinear terms $u_{x} u_{x x}$ and $u_{x x}^{2}$ having the value

$$
\begin{aligned}
& A(x, k)=\sum_{r=0}^{k} \frac{\partial U(x, r)}{\partial x} \frac{\partial^{2} U(x, k-r)}{\partial x^{2}}, \\
& B(x, k)=\sum_{r=0}^{k} \frac{\partial^{2} U(x, r)}{\partial x^{2}} \frac{\partial^{2} U(x, k-r)}{\partial x^{2}} .
\end{aligned}
$$

The few nonlinear terms are as follows

$$
\begin{aligned}
& A(0)=U_{x}(0) U_{x x}(0) \\
& A(1)=U_{x}(0) U_{x x}(1)+U_{x}(1) U_{x x}(0) \\
& A(2)=U_{x}(0) U_{x x}(2)+U_{x}(1) U_{x x}(1)+U_{x}(2) U_{x x}(0)
\end{aligned}
$$


and so on.

$$
\begin{aligned}
& B(0)=U_{x x}^{2}(0) \\
& B(1)=2 U_{x x}(0) U_{x x}(1) \\
& B(2)=2 U_{x x}(0) U_{x x}(2)+U_{x x}^{2}(1)
\end{aligned}
$$

and so on.

From the relationship in (5.13), (5.14), we obtain

$$
\begin{aligned}
u(x, 0)= & t x^{2}, \\
u(x, 1)= & E^{-1}\left[v^{2} E\left[x^{2} \frac{\partial}{\partial x} A(x, 0)-x^{2} B(x, 0)-u(x, 0)\right]\right]=-\frac{t^{3}}{3 !} x^{2}, \\
u(x, 2)= & E^{-1}\left[v^{2} E\left[x^{2} \frac{\partial}{\partial x} A(x, 1)-x^{2} B(x, 1)-u(x, 1)\right]\right]=\frac{t^{5}}{5 !} x^{2}, \\
u(x, 3)= & E^{-1}\left[v^{2} E\left[x^{2} \frac{\partial}{\partial x} A(x, 2)-x^{2} B(x, 2)-u(x, 2)\right]\right]=-\frac{t^{7}}{7 !} x^{2}, \\
& \ldots,
\end{aligned}
$$

and so on.

Which in closed form gives exact solution

$$
\begin{aligned}
u(x, t) & =\sum_{k=0}^{\infty} U(x, k)=x^{2}\left(t-\frac{t^{3}}{3 !}+\frac{t^{5}}{5 !}-\frac{t^{7}}{7 !}+\ldots\right) \\
& =x^{2} \sin t .
\end{aligned}
$$

which is an exactly the same solution obtained by Adomian decomposition method [6] and Homotopy perturbation transform method [7] for the same test problem.

\section{Discussion of Results}

We present in this section to discuss our obtained results in comparison with their associated exact forms. Figures 1-2-3 are 2D plots of the approximate solution and exact solution where $x=y=0.5$ and $t \in[0.1]$ for Example 1 and $x=0.5, t \in[0.1]$ for Examples 2-3. The tables 1-2-3 are Comparison of the absolute errors for the obtained results and the exact solution for Examples 1-2-3, where $x, y, t \in[0.1]$ and $n=3$.

We define $E_{n}(X, t)$ to be the absolute error between the exact solution $u(X, t)$ and $n$-term approximate solution by EPDTM

$$
\varphi_{n}(X, t)=\sum_{k=0}^{n-1} u(X, k)
$$

as follows

$$
E_{n}(X, t)=\left|u(X, t)-\varphi_{n}(X, t)\right|
$$




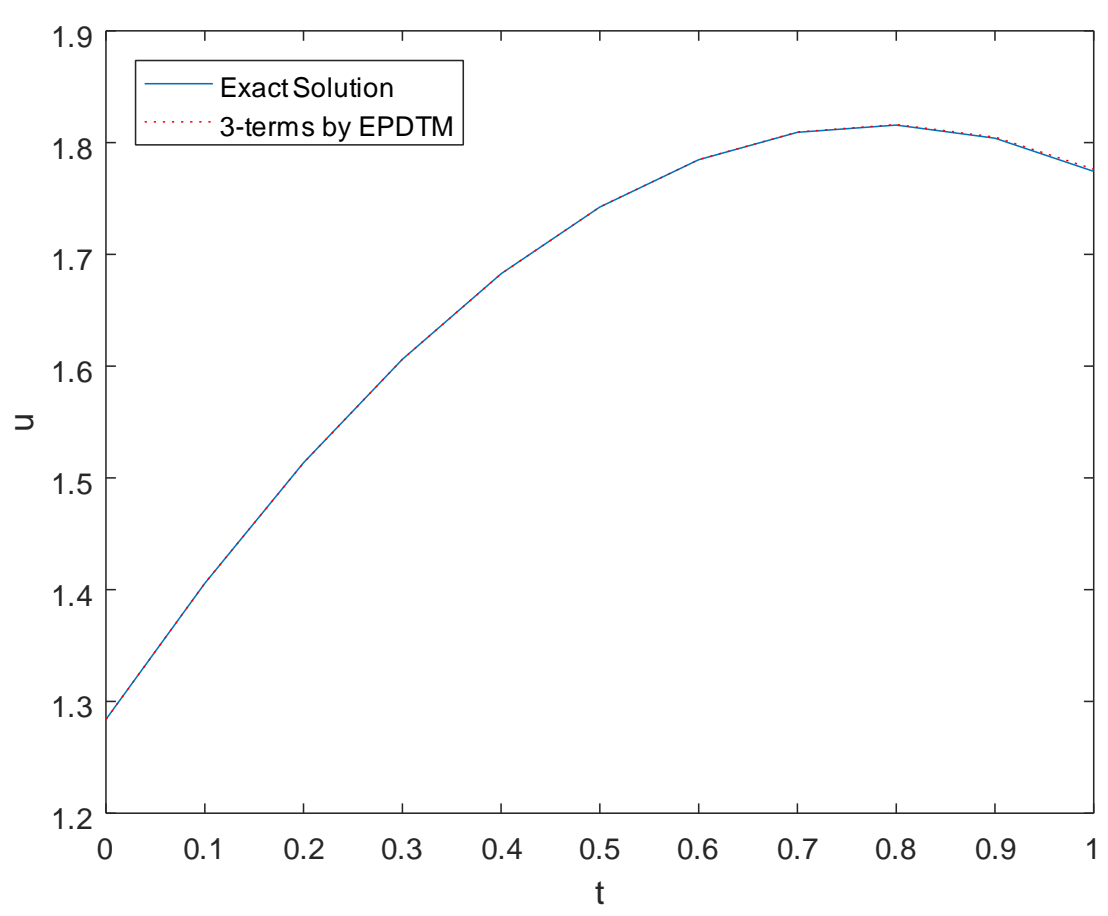

Figure 1. The behavior of the exact solution and the 3terms approximate solution for Example 1 when $x=y=0.5$.

TABLE 1. Comparison of the absolute errors for the obtained results and the exact solution for Example 1, where $n=3$

\begin{tabular}{lcccc}
\hline$t / x, y$ & 0.1 & 0.3 & 0.5 & 0.7 \\
\hline 0.1 & $1.4226 \times 10^{-9}$ & $1.5411 \times 10^{-9}$ & $1.8085 \times 10^{-9}$ & $2.2991 \times 10^{-9}$ \\
0.3 & $1.0648 \times 10^{-6}$ & $1.1535 \times 10^{-6}$ & $1.3536 \times 10^{-6}$ & $1.7208 \times 10^{-6}$ \\
0.5 & $2.3382 \times 10^{-5}$ & $2.5330 \times 10^{-5}$ & $2.9725 \times 10^{-5}$ & $3.7787 \times 10^{-5}$ \\
0.7 & $1.8000 \times 10^{-4}$ & $1.9499 \times 10^{-4}$ & $2.2882 \times 10^{-4}$ & $2.9089 \times 10^{-4}$ \\
0.9 & $8.2963 \times 10^{-4}$ & $8.9872 \times 10^{-4}$ & $1.0547 \times 10^{-3}$ & $1.3407 \times 10^{-3}$ \\
\hline
\end{tabular}




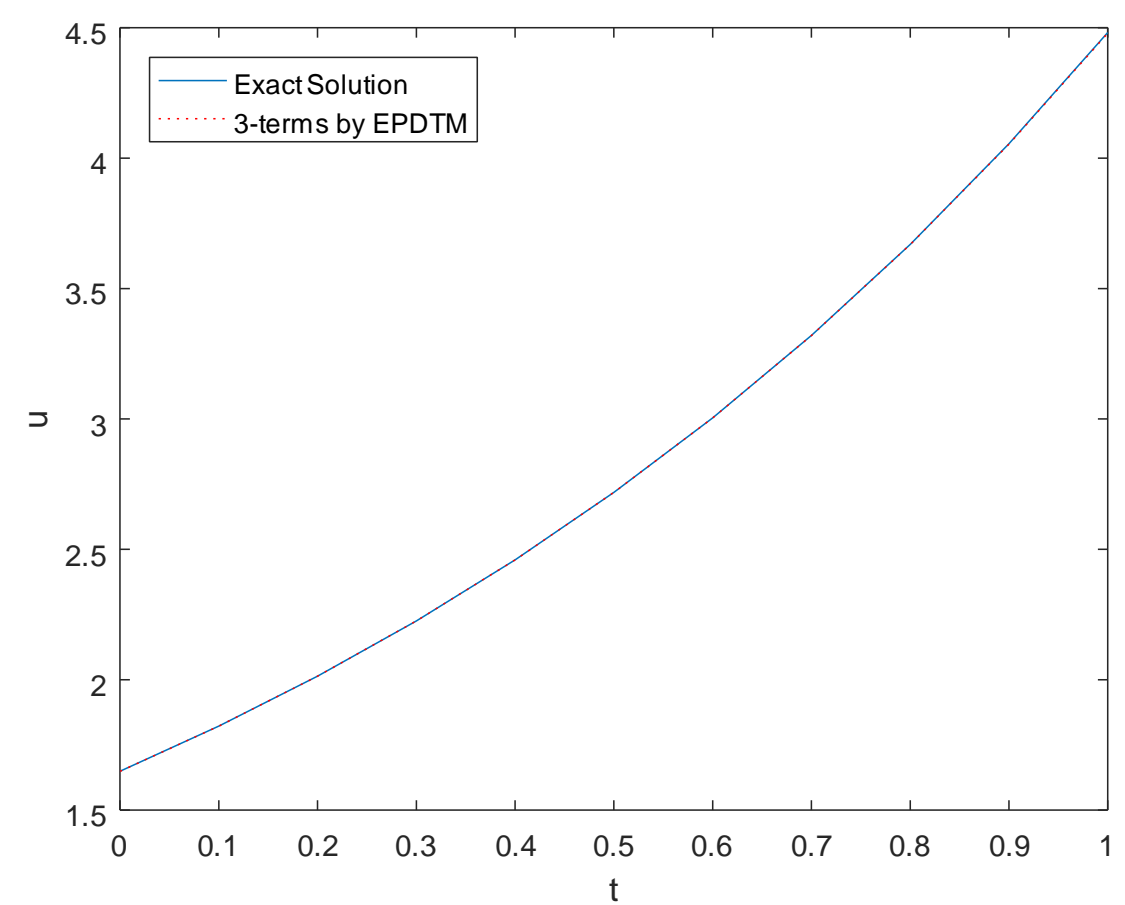

FiguRE 2. The behavior of the exact solution and the 3terms approximate solution for Example 2 when $x=0.5$.

TABle 2. Comparison of the absolute errors for Example 2, where $\mathrm{n}=3$

\begin{tabular}{lcccc}
\hline$t / x, y$ & 0.1 & 0.3 & 0.5 & 0.7 \\
\hline 0.1 & $1.5572 \times 10^{-9}$ & $1.9019 \times 10^{-9}$ & $2.3230 \times 10^{-9}$ & $2.8373 \times 10^{-9}$ \\
0.3 & $1.1688 \times 10^{-6}$ & $1.4276 \times 10^{-6}$ & $1.7436 \times 10^{-6}$ & $2.1297 \times 10^{-6}$ \\
0.5 & $2.5810 \times 10^{-5}$ & $3.1525 \times 10^{-5}$ & $3.8504 \times 10^{-5}$ & $4.7029 \times 10^{-5}$ \\
0.7 & $2.0036 \times 10^{-4}$ & $2.4472 \times 10^{-4}$ & $2.9890 \times 10^{-4}$ & $3.6507 \times 10^{-4}$ \\
0.9 & $9.3372 \times 10^{-4}$ & $1.1404 \times 10^{-3}$ & $1.3929 \times 10^{-3}$ & $1.7013 \times 10^{-3}$ \\
\hline
\end{tabular}




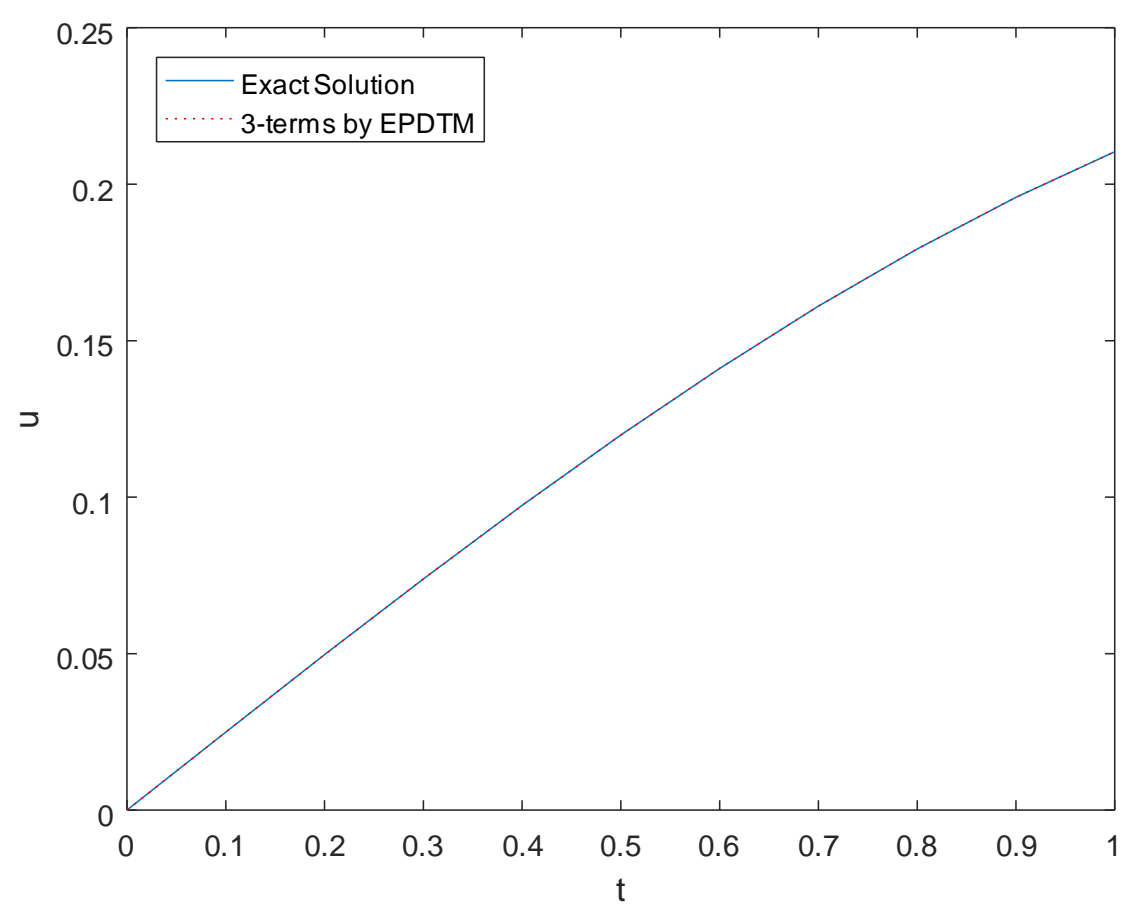

FiguRE 3. The behavior of the exact solution and the 3terms approximate solution for Example 3 when $x=0.5$.

TABle 3. Comparison of the absolute errors for Example 3, where $\mathrm{n}=3$

\begin{tabular}{lclll}
\hline$t / x, y$ & \multicolumn{1}{c}{0.1} & 0.3 & \multicolumn{1}{c}{0.5} & 0.7 \\
\hline 0.1 & $1.9839 \times 10^{-13}$ & $1.7855 \times 10^{-12}$ & $4.9596 \times 10^{-12}$ & $9.7209 \times 10^{-12}$ \\
0.3 & $4.3339 \times 10^{-10}$ & $3.9005 \times 10^{-9}$ & $1.0835 \times 10^{-8}$ & $2.1236 \times 10^{-8}$ \\
0.5 & $1.5447 \times 10^{-8}$ & $1.3903 \times 10^{-7}$ & $3.8618 \times 10^{-7}$ & $7.5692 \times 10^{-7}$ \\
0.7 & $1.6229 \times 10^{-7}$ & $1.4606 \times 10^{-6}$ & $4.0574 \times 10^{-6}$ & $7.9524 \times 10^{-6}$ \\
0.9 & $9.3840 \times 10^{-7}$ & $8.4456 \times 10^{-6}$ & $2.3460 \times 10^{-5}$ & $4.5982 \times 10^{-5}$ \\
\hline
\end{tabular}




\section{Conclusion}

In this work, we used a mixed Elzaki transform and the projected differential transformation method, the advantage is providing an analytical approximation of the solution, usually an exact solution, in a fast convergent sequence for nonlinear wave-like equations with variable coefficients. EPDTM can be performed very easily is more efficient and reliable compared to the most known techniques (Adomian decomposition and homotopy perturbation) as in [6], 7]. In addition, EPDTM is faster than ADM-HPM to solve this type of equation and can be applied to other nonlinear partial differential equations.

\section{REFERENCES}

[1] A. K. Alomari, M. S. M. Noorani, R. Nazar, Solutions of Heat-Like and Wave-Like Equations with Variable Coefficients by Means of the Homotopy Analysis Method, Chinese Physics Letters, 25(2) (2008), 589.

[2] A. Atangana, E. Alabaraoye, Exact Solutions Fractional Heat-Like and WaveLike Equations with Variable Coefficients, Open Access Scientific Reports, http://dx.doi.org/10.4172/scientificreports.633.

[3] A. Aslanov, Homotopy Perturbation Method for Solving Wave-Like Nonlinear Equations with Initial-Boundary Conditions, Discrete Dynamics in Nature and Society, Article ID 534165 (2011), 10 pages.

[4] T. M. Elzaki \& S. M. Elzaki , Application of New Transform "Elzaki Transform" to Partial Differential Equations, Global Journal of Pure and Applied Mathematics, ISSN 0973-1768, Number 1 (2011), pp. 65-70.

[5] T. M. Elzaki and S. M. Elzaki and E. A. Elnour, On the New Integral Transform "Elzaki Transform" Fundamental Properties Investigations and Applications, Glo. J. Math. Sci., 4, (2012), 1-13.

[6] M. Ghoreishi, A.I.B. Ismail, N.H.M.Ali, Adomain decomposition method for nonlinear wave- like equation with variable coefficients, Applied Mathematical Sciences, Vol. 4 ,No. 49, 2431- 2444, 2010.

[7] V.G. Gupta and S. Gupta, Homotopy perturbation transform method for solving nonlinear wave- like equations of variable coefficients, Journal of Information and Computing Science, Vol. 8, No.3,163-172, 2013.

[8] B. Jang, Solving linear and nonlinear initial value proplems by the projected differential transform method. Computer Physics Communications 181, 848-854, (2010).

[9] A. Kilicman and H. Eltayeb, A note on Integral transform and Partial Differential Equation, Applied Mathematical Sciences, 4(3), (2010), PP.109-118. Mathematical Theory and Modeling, ISSN 2224-5804 (Paper), Vol.2, No.4, 2012.

[10] S. Momani, Analytical approximate solution for fractional heat-like and wave-like equations with variable coefficients using the decomposition method, Applied Mathematics and Computation, 165 (2005), 459-472.

[11] A.M. Wazwaz and A. Gorguis, Exact solutions for heat-like and wave-like equations with variable coefficients, Applied Mathematics and Computation, 149 (2004), 15-29.

[12] F. Yin, J. Song, X. Cao, A General Iteration Formula of VIM for Fractional Heat- and Wave-Like Equations, Journal of Applied Mathematics, Article ID 428079 (2013), 9 pages. 
${ }^{1}$ A. Khalouta, Laboratory of Fundamental and Numerical Mathematics, Departement of Mathematics, Faculty of Sciences. University of Sétif 1 , 19000 Sétif, Algeria.

E-mail address: nadjibkh@yahoo.fr

2 A. Kadem, Laboratory of Fundamental and Numerical Mathematics., Departement of Mathematics, Faculty of Sciences. University of Sétif 1, 19000 SÉtif, Algeria.

E-mail address: abdelouahabk@yahoo.fr 